\title{
Improved Reversible Mapping from Color to Gray
}

\author{
Ricardo L. de Queiroz \\ Dept. Engenharia Elétrica \\ Universidade de Brasília \\ Brasília, Brazil \\ queirozeieee.org
}

\begin{abstract}
In the reversible conversion of color images to gray ones, colors are mapped to textures and from the textures the receiver can recover the colors, i.e. one may print a color image with a black and white printer and, at a later time, recover colors. Such a method was originally devised using the wavelet transform and replacing high-frequency subbands by subsampled chrominance planes. Here, we propose to improve the original method thorugh the use of a largely redundant representation of the chrominance, with multiple embedding into subbands of a general subband transform. Our results point to minimization of the variance of the error caused by noise when the chrominance is replicated into many subbands and not linearly combined. Derived noise bounds guide us on how many subbands to embed the chrominance. Experimental results were carried for multiple noise levels, models, transform sizes, and number of embedding subbands, demonstrating the theoretical analysis and the method's potential.
\end{abstract}

\section{INTRODUCTION}

Recently, an innovative method to convert color images into gray ones was introduced [1]. Its key feature was to be reversible. The color image is converted to gray scales and one can retrieve the colors from the gray image at a later time. A user with an electronic color document may only have easy access to monochrome office laser printers, or to a fax machine. Thus, the color document is first converted to gray scales, then to black and white, before printing (or faxing). Later on, the user might scan (receive) the black and white document, recover the monochrome (gray) image, process it, and retrieve the colors. The process is illustrated in Fig. 1.

We map colors to high frequency textures. By analysing the textures, the user retrieves the colors for each region. In the originally proposed method [1] one has to: (i) Convert the color image to some luminance-chrominance color space such as YCbCr or CIELab [2]. (ii) Apply the discrete wavelet transform (DWT) to the luminance channel [3],[4]. (iii) Spatially reduce (subsample) the 2 chrominance planes by a factor of 2 in each direction. (iv) Replace the high-frequency wavelet subbands [4](HL and LH) by the 2 chrominance planes. (v) Apply the inverse DWT yielding a texturized gray image (because of the embedded chrominance planes acting as subband coefficients). (vi) Scale, halftone [5] and print (or transmit) the texturized gray image. The method is illustrated in Fig. 2.

In order to recover the color from the gray texturized image, as illustrated in Fig. 3, one has to: (i) Scan (receive) the image

This work was supported in part by a grant from HP Brasil.

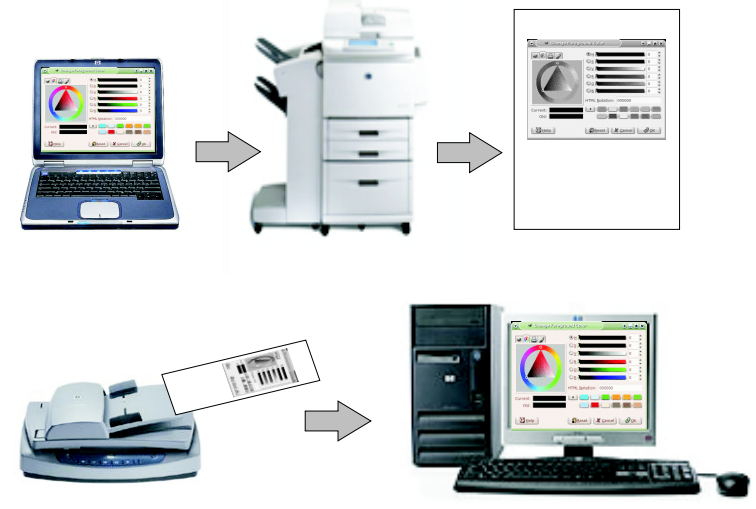

Fig. 1. Illustration of the color to gray conversion application.

and convert it to gray scale. (ii) Apply the DWT to the gray image. (iii)Retrieve the high frequency subbands and assign them as chrominance planes. (iv) Set to zero the subbands which were used to embed chrominance. (v) Apply an inverse DWT to the resulting subbands, yielding the luminance plane. (vi) Spatially increase (up-sample) the 2 chrominance planes by a factor of 2 in each direction. (vii) Convert the luminancechrominance planes back into RGB.

In essence, colors are converted to strong high-frequency patterns. For the normal user they look like halftones or similar textures which blend with the objects. Examples are shown in Fig. 4. The method works so well because the embedding is natural, since the colors match the objects. If we coded the chrominance and embedded the binary information into the image using any typical image watermarking technique [6], it would probably not work. The encoded information does not correlate with the image contents. In order to avoid artifacts one would have to make it subtle or invisible. Then, it is likely that the information would be removed by the halftoning, printing and scanning processes. If we make the embedded information strong enough to survive halftoning it would likely cause artifacts. At the moment, despite the work in this direction [7]-[15], we do not have reliable non-intrusive watermarking methods for printed images, without controlling printer characteristics such as the halftone algorithm or the laser beam intensity. The proposed method produces strong but pleasant patterns, making it an excellent candidate for the 

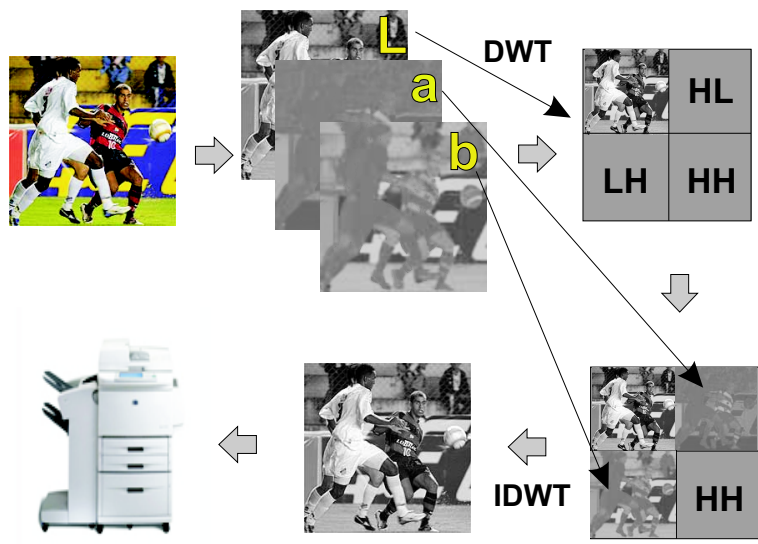

Fig. 2. The color to gray conversion method. The luminance is decomposed into subbands through a wavelet transform. The high pass bands are replaced by the chrominance planes and the image is inverse transformed generating a texturized gray image which conveys the color information.

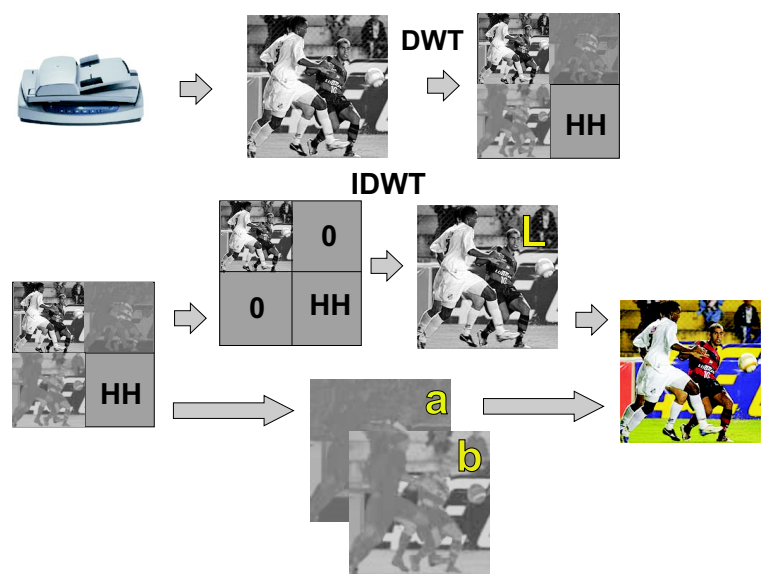

Fig. 3. The color recovery method. After scanning the image, the texturized image undergoes a DWT, the chrominance planes are extracted, the corresponding subbands are zeroed, and an inverse DWT is carried on the result, yielding the luminance channel. The luminance and chrominance channels are used to recover the color image.

application.

\section{EMBEDDING CHROMINANCE INTO SUBBANDS}

The method presented in the previous section is simplistic. If the gray image was to be scaled up, halftoned and faxed, it would work very well, allowing the recovery of vivid colors. If the image is to be printed, the noise and errors are so intense that the simple method presented would not work. Any minimal shift of a pixel or rotations of one degree may completely impair the color recovery [1]. As a result, in the original work [1], some non-linear redundancy was introduced in the wavelet domain to prevent sign inversion and reduce noise sensitivity, with some success.

We propose to use a more robust redundancy scheme to cope with noise. Indeed, we propose to:

- Use general $M$-band subband transforms [3] rather than the DWT, allowing the use of the discrete cosine transform (DCT) [16] or general lapped transforms [17],[18].

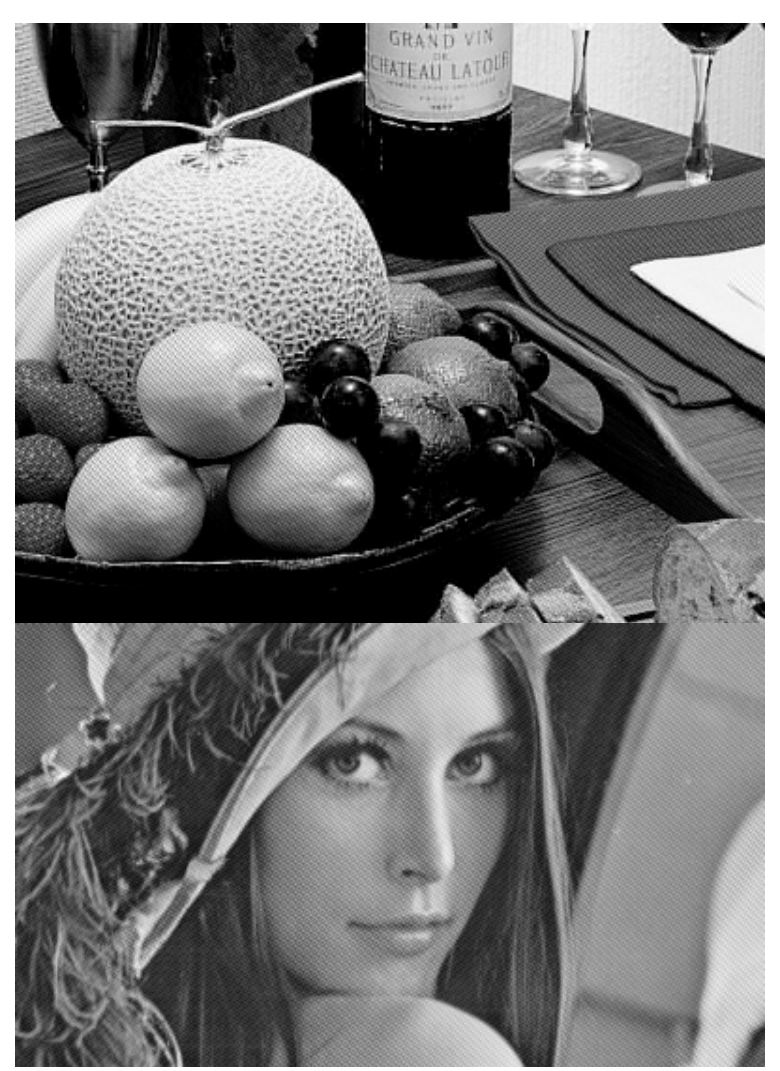

Fig. 4. Zoom of three example texturized images using the proposed method, with a $4 \times 4$ DCT and $N=6$. The high frequency patterns tend to interfere with sampling grids resulting in annoying artifacts when displaying those texturized images at lower resolution. Changing the viewing resolution may change the artifacts. PDF conversion also causes artifacts.

- Embed the chrominance planes into a linear combination of multiple subbands per chrominance channel, as illustrated in Fig. 5.

- Decide upon the redundancy based on a signal to noise model analysis of the embedding mechanisms.

The questions that need to be answered before we apply such a method are:

- (a) What subband transform should we use?

- (b) How many channels $M$ should it have?

- (c) How many subbands $N_{s}$ should we use per chrominance?

- (d) How do we decide upon the linear combination of the subband, i.e. how do we distribute the chrominance information onto the $N_{s}$ channels?

As for the transform (a) we can use lapped transforms such as the lapped orthogonal transform (LOT), the modulated (extended) lapped transform (MLT or ELT) [17], or even the DCT [16]. All these $M$-band subband transforms will be similar to full-tree discrete wavelet packets, in the end. As a note, we do want to grow the full tree, i.e. to use a full $M$-band transform, since we want more subbands where to embed the chrominance signal. Hence, for the work here, what is important is to have an $M$-band subband transform. We have run tests that indicate that the subband transforms, here proposed, outperform the previous method in [1] which is based on the DWT. Test results also show that the DCT 


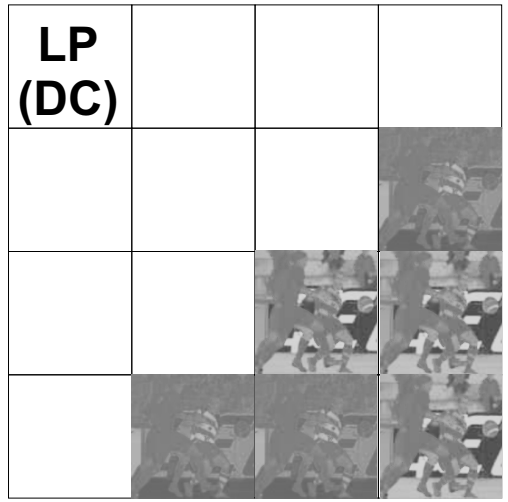

Fig. 5. Illustration of the proposed method, showing an example of the subbands of a $4 \times 4$ transform, indicating the low-pass or DC band. In this example, 3 copies of each chrominance channel were alternately embedded into the highest frequency subbands.

slightly outperforms the other lapped transforms tested. These test results are in the full version [19] of this paper along with many theoretical results. The choice on the number of bands (question b), we believe to be a function of $N_{s}$. We do not want to use too many subbands to carry chrominance information, because it may impair the quality of the luminance information. For example, one can use a $4 \times 4$-band $(M=16)$ transform to embed up to 6 bands $\left(N_{s}=3\right)$, no more than 10 , and higher numbers may be accommodated into an $8 \times 8$ transform. Note that the larger $M$ is, the better the luminance quality, but the worst the chrominance representation since the chrominance planes must be sub-sampled accordingly to fit the subbands. Hence, we want $M$ to be as small as possible as long as $M$ is substantially larger than $N_{s}$. A detailed analysis on deciding upon $N_{s}$ will be carried later on and questions (c,d) will be answered in the following sections.

The chrominance channels can be expressed as the linear combination of the information into a plurality of subbands. However, rather than linearly combining the information into many subbands it is better to replicate each chrominance plane onto many subbands (no combining), i.e. simply replicate each of the chrominance planes into as many subbands as desired, without bothering to do any linear combination between them. The support for this assertion comes from a Theorem that deals with multiple description methods, whose proof involves some algebra and is left to a Journal version of this paper.

Let $N=2 N_{s}$ (equal number of embedded subbands for each of the chrominances). The more subbands we use, the better the chrominance reproduction, but when we use one subband to embed a chrominance plane or its replica, we actually erase the luminance in that subband. So, as the distortion decreases because we improve chrominance reproduction, at the same time distortion also increases because luminance reproduction suffers. Without loss of generality, let us number the $M$ subbands one-dimensionally, arranging them such that the embedded luminance subbands would be the last $N$. More specifically, if we have already $N$ subband embedded, is it worthy to embed two more, one for each chrominance channel? Assume for the moment that the printing-scanning process causes an image distortion that is stationary, white and has variance (energy) $\sigma_{n}^{2}$. We can show (in the Journal version of this paper) that it is only advantageous to embed chrominance into two more subbands in terms of SNR if

$$
\sigma_{n}^{2}>\frac{N(N+2)}{8 M}\left(\sigma_{s_{M-N-1}}^{2}+\sigma_{s_{M-N-2}}^{2}\right) .
$$

where $\sigma_{s_{M-N-1}}^{2}$ and $\sigma_{s_{M-N-2}}^{2}$ are the variances of the next two luminance subbands that will be replaced by those further two chrominance replicae. The above condition tells us that if the textured image suffers small noise levels, it is not advantageous to add chrominance redundancy in expense of luminance quality. In the extreme case of noise free transmission, one can perfectly recover both chrominance channels with only two subbands. No redundancy is necessary. As the noise increases, the chrominance information disappears as noise corrupts the textures, and it becomes necessary to add redundancy (more subbands) to protect the channel. This equation also tells us that, as $N$ grows, it becomes harder and harder to get any benefit from embedding even more subbands. Of course, it all depends on the variance of the luminance subbands that will be discarded. The more intense their energy, the more intense the noise has to be to make it worthy embed more subbands.

\section{EXPERIMENTS}

The condition in (1) is image dependent and hard to compute. Furthermore, since we do not have reliable methods to measure the noise, it is not trivial to determine $\sigma_{n}^{2}$. We have assumed the print-scan distortion to be additive white Gaussian noise. It is a reasonable model, supported by a number of reasons. Firstly, geometric distortions, caused by paper warping and misplacement, halftoning and other sources contribute to the overall image distortion. However, as we apply corrections to these distortions, filter the halftone, and so on, the resulting noise is not that correlated to the image any longer. Secondly, the quality of reconstruction is heavily dependent on the implementation of the geometric corrections, e.g. the interpolation in the affine transform correction mechanism. Different implementations would have large impact on the final result, nevertheless they are unrelated to this work. Furthemore, a study on modelling the print-scan noise will be presented elsewhere. At last, it is not practical to perform a large batch of tests to determine parameters if we had to print and scan the images every time. Hence, we will model the noise as additive. We can also improve the model incorporating halftones:

- Scale (interpolate) the gray image up by a factor of $K$ in each direction.

- Halftone the resulting image using error diffusion [5].

- Filter the bi-level image using a short Gaussian filter to soften the dots borders.

- Add zero-mean white noise with variance $\sigma_{p}^{2}$.

- Downsample by averaging $K \times K$-pixel blocks of the noisy, filtered, halftoned image.

In this way, noise also comes from the halftoning, which is reversed by simple averaging. If $K=1$ there is no inverse halftoning process. In oder to account for geometric distortions such as rotation and shearing caused by printing, and other 


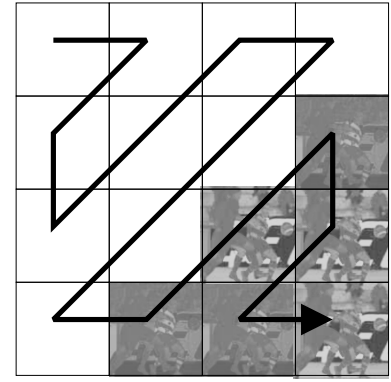

Fig. 6. Zigzag ordering of subbands.

physical effects of putting ink on paper, we include additive noise to the halftoned image. We first filtered it to further distort the bitmap. This contrasts with the work in [1] which used no noise model.

In order to verify our model we carried experiments for a few color images. Subband ordering following the zigzag path as shown in Fig. 6 was used. The subsampled chrominance planes are alternately embedded into the last high frequency subbands according to the zigzag path, as shown in Fig. 6. We used two transforms: the $4 \times 4$ DCT $(M=16)$ and the $8 \times 8$ DCT $(M=64)$. Lapped transforms and other values of $M$ could be used as well, but these two transforms are sufficient for this paper's analytical purposes.

In a first set of tests, the RGB image was converted into a gray-scales image, embedding the chrominance into the last $N$ out of $M$ subbands. Zero-mean white noise with variance $\sigma_{n}^{2}$ was added to the gray image, interfering with the textures. The color image is, then, retrieved, and we compute the mean squared error between the two RGB images. The results for two different images, and various values of $N, M$ and $\sigma_{n}^{2}$ are presented in Table I.

From the results we can see a clear pattern that confirms our theoretical results. For lower noise values, as we increase $N$, the MSE increases. Whenever the noise is more intense, as we increase $N$, the MSE actually decreases. We can see that for image "wine" the noise variance bound in (1) lies between 16 and 32 for all values of $N$ tested, for $M=16$. The same bounds serve for $M=64$ and $N \leq 6$. For $N>6, \sigma_{n}^{2}$ seems to be above 32 and under 64. Image "Lena" has less details and not as many extreme colors as image "wine" and, for that, the noise bounds are much lower because the high-frequency subband variances are smaller. For $M=16$ and image "Lena", the bound of $\sigma_{n}^{2}=6$ seems to apply to all values of $N$ tested. A similar value applies to the $M=64$ case. For a range of noise values the relation between $N$ and the MSE is not monotonic but around $\sigma_{n}^{2}=10$ the behaviour becomes monotonic once again. In these experiments, we modeled the noise as uncorrelated and additive. In reality there is much more to it, but the model is a beginning.

The noise levels, however, were not overestimated. In order to improve noise estimation and simulate the printing and scanning process, we applied the following steps: Tests with the print simulation (adding noise with variance $\sigma_{p}^{2}$ to the halftone) were carried and results for image "wine" and $4 \times 4$ DCT are presented in Table II.
TABLE I

MSE RESULTS (IN RGB SPACE) FOR EMBEDDING CHROMINANCE INTO MULTIPLE SUBBANDS OF THE DCT FOR VARIOUS NOISE LEVELS $\sigma_{n}$ AND NUMBER OF BANDS $N$.

\begin{tabular}{|c|c|c|c|c|c|c|}
\hline \multicolumn{7}{|c|}{$4 \times 4$ DCT - Image "wine" } \\
\hline $\bar{N}$ & \multicolumn{6}{|c|}{$\overline{\sigma_{n}^{2}}$} \\
\hline & 0 & 4 & 8 & 16 & 32 & 64 \\
\hline 2 & 76.8 & 100.7 & 172.0 & 437.1 & 1387.4 & 4350.4 \\
\hline 4 & 101.8 & 124.1 & 185.8 & 440.5 & 1333.6 & 4128.9 \\
\hline 6 & 140.5 & 160.5 & 220.5 & 447.3 & 1287.8 & 3886.0 \\
\hline 10 & 259.3 & 276.1 & 326.6 & 514.2 & 1203.6 & 3489.2 \\
\hline \multicolumn{7}{|c|}{$8 \times 8$ DCT - Image "wine" } \\
\hline$N$ & \multicolumn{6}{|c|}{$\sigma_{n}^{2}$} \\
\hline & 0 & 4 & 8 & 16 & 32 & 64 \\
\hline 2 & 118.8 & 143.1 & 212.5 & 495.4 & 1497.9 & 4519.7 \\
\hline 4 & 121.7 & 144.6 & 218.7 & 487.0 & 1460.6 & 4442.5 \\
\hline 6 & 125.4 & 148.8 & 219.5 & 481.6 & 1507.4 & 4424.3 \\
\hline 10 & 135.2 & 157.2 & 226.9 & 484.7 & 1455.6 & 4385.9 \\
\hline 16 & 154.4 & 175.1 & 238.3 & 489.6 & 1381.0 & 4264.9 \\
\hline \multicolumn{7}{|c|}{$4 \times 4$ DCT - Image "Lena" } \\
\hline$N$ & \multicolumn{6}{|c|}{$\sigma_{n}^{2}$} \\
\hline & 0 & 4 & 8 & 16 & 32 & 64 \\
\hline 2 & 22.6 & 50.3 & 132.6 & 449.7 & 1599.4 & 4793.9 \\
\hline 4 & 24.4 & 50.1 & 126.6 & 424.5 & 1480.1 & 4526.4 \\
\hline 6 & 26.3 & 50.0 & 119.7 & 395.6 & 1387.7 & 4236.2 \\
\hline 10 & 35.2 & 55.1 & 113.5 & 342.2 & 1189.9 & 3730.3 \\
\hline \multicolumn{7}{|c|}{$8 \times 8$ DCT - Image "Lena" } \\
\hline$N$ & \multicolumn{6}{|c|}{$\overline{\sigma_{n}^{2}}$} \\
\hline & 0 & 4 & 8 & 16 & 32 & 64 \\
\hline 2 & 38.2 & 65.8 & 153.5 & 487.4 & 1673.6 & 4935.1 \\
\hline 4 & 38.4 & 66.5 & 155.1 & 476.5 & 1676.4 & 4901.5 \\
\hline 6 & 38.6 & 66.9 & 147.7 & 467.3 & 1617.3 & 4933.2 \\
\hline 10 & 39.1 & 66.6 & 146.6 & 458.7 & 1601.9 & 4734.5 \\
\hline 16 & 39.9 & 65.5 & 139.7 & 433.8 & 1481.8 & 4459.5 \\
\hline
\end{tabular}

The halftoning process causes so much degradation that all noise values $\sigma_{p}^{2}$ yield decaying MSE for increasing $N$. Figure 7 shows a zoom of part of the image that would be seen by a scanner, i.e. right after scaling by $K$, error diffusion halftoning, Gaussian blurring and white noise corruption. The image is nearly binary and very noisy. It is interesting that this image still carries color information. The noise is so intense that the more subbands we use to protect the chrominance the better. Of course there is a limit that is not shown in the tables. If we extend the results in Table II for the case $K=4$ and $\sigma_{p}=0$, we would obtain the MSE results shown in Fig. 8. Note that as $N$ increases too much, we run out of low-energy subbands where to embed the chrominance copies. In fact, at some point, the subband variances in (1) become too high, and that compounds with the fact that the term grows with $N^{2}$, limiting the effectiveness of further embedding. The minimum is reached at $N=10$, which corresponds to the last four diagonal line of subbands. Of course, there will be some blocking artifacts in the luminance channel, but that, apparently, is compensated by the improvements in chrominance recovery.

The results and their associated discussion, in this section, serve to point to the effectiveness of the method of linear redundancies in the subband domain. In typical scenarios, embedding chrominance into more subbands may improve the reconstructed image quality. As an example, the recovered color image for $N=10$ in a $4 \times 4$ DCT, $K=4, \sigma_{p}^{2}=25$ is shown in Fig. 9. The colors are reasonably vivid, but the reader 
TABLE II

MSE RESULTS (IN RGB SPACE) FOR EMBEDDING CHROMINANCE INTO MULTIPLE SUBBANDS, AFTER SCALING, HALFTONING AND SIMULATED PRINTING.

\begin{tabular}{|c|c|c|c|c|}
\hline \multicolumn{5}{|c|}{$4 \times 4$ DCT - Image "wine" } \\
\hline$N$ & \multicolumn{4}{|c|}{$\overrightarrow{\sigma_{p}^{2}}$} \\
\hline & 0 & 10 & 25 & 50 \\
\hline \multicolumn{5}{|c|}{$K=2$} \\
\hline 2 & 1730.9 & 1761.9 & 1928.7 & 2547.9 \\
\hline 4 & 1607.9 & 1641.9 & 1805.3 & 2352.4 \\
\hline 6 & 1402.5 & 1432.5 & 1578.8 & 2152.2 \\
\hline \multicolumn{5}{|c|}{$K=4$} \\
\hline 2 & 1194.5 & 1206.2 & 1251.7 & 1424.6 \\
\hline 4 & 1132.4 & 1139.6 & 1186.9 & 1351.3 \\
\hline 6 & 1016.3 & 1020.2 & 1069.4 & 1209.1 \\
\hline \multicolumn{5}{|c|}{$K=6$} \\
\hline 2 & 1132.5 & 1136.2 & 1156.7 & 1243.7 \\
\hline 4 & 1081.5 & 1086.3 & 1105.7 & 1183.6 \\
\hline 6 & 975.4 & 978.7 & 998.5 & 1070.9 \\
\hline
\end{tabular}

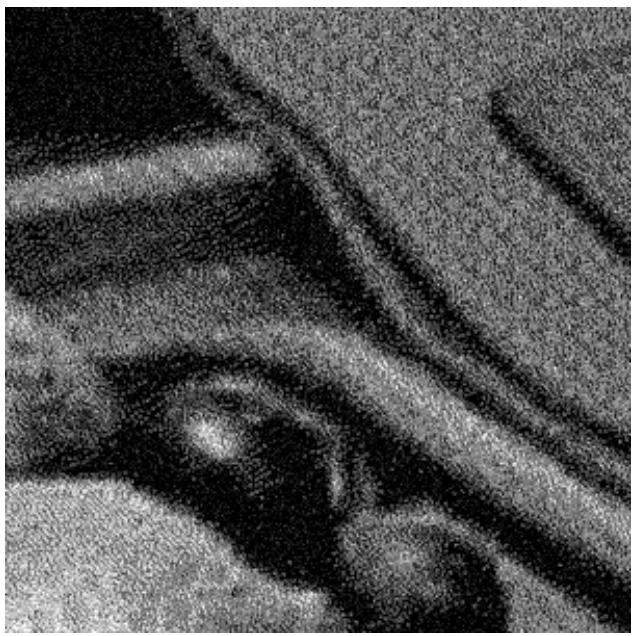

Fig. 7. Zoomed portion of the gray (near binary) image that would be seen by a scanner, after scaling by $K=4$, halftoning using error diffusion, blurring with a Gaussian filter and white noise corruption with $\sigma_{p}^{2}=25$.

should take into consideration that the image is a starting point that can be further corrected using photo editing software tools. Furthermore, it has to be put in the right perspective the fact that we start with a black and white noisy halftone image shown in Fig. 7 (enlarged portion) to get to the color image. With that in mind the results can be considered very good.

\section{CONCLUSIONS}

In this paper, we proposed a method for reversible conversion from color images to gray ones based on redundant color information embedded into subbands of an $M$-band subband transform. Among the contributions, we propose to use a general subband transform rather than wavelets, since it generates more subbands, and the insertion of color information with strong linear redundancy. We have shown that the ideal distribution of the chrominance energy among many subbands is to replicate the chrominance plane for each subband. We also have calculated the SNR performance of the method and the bounds that limit the effectiveness of chrominance embedding, as well as the amount of redundancy.

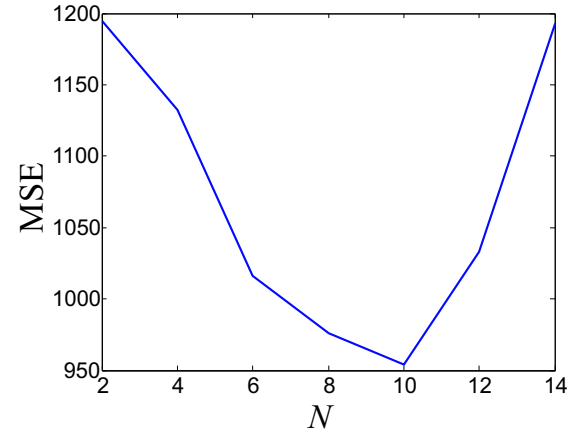

Fig. 8. MSE plot for $N=10$ in a $4 \times 4$ DCT embedding framework. Printing simulation used error diffusion, $K=4$ and no additive noise.

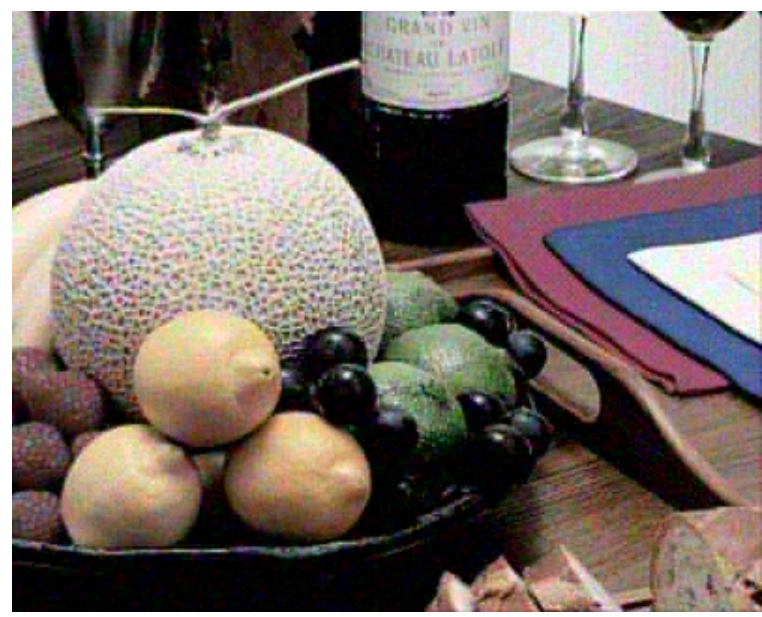

Fig. 9. The recovered color image for $N=10$ in a $4 \times 4$ DCT framework is shown at the top. Printing simulation includes error diffusion, Gaussian filtering, scaling by $K=4$, and noise with variance $\sigma_{p}^{2}=25$.

Redundant subband embedding of chrominance planes distorts luminance but improves the robustness of the color information. The bounds tell us that if the noise level is high enough one should spare more luminance subbands to protect the chrominance channel. The printing-scanning path is simulated and our results show that the noise is high enough to warrant the embedding of color into many subbands. In fact, the results show that our method is effective.

\section{REFERENCES}

[1] R. L. de Queiroz and K. Braun, "Color to gray and back: color embedding into textured gray images," IEEE Trans. on Image Processing, Vol. 15, No 6, pp. 1464-1470, June 2006.

[2] R. W. G. Hunt, The Reproduction of Color, Fountain Press, Toolworth, England, 2000.

[3] M. Vetterli and J. Kovacevic, Wavelets and Subband Coding, Englewood Cliffs, NJ: Prentice-Hall, 1995.

[4] G. Strang and T. Nguyen, Wavelets and Filter Banks, Wellesley, MA: Wellesley-Cambridge, 1996.

[5] P. G. Roetling and R. P. Loce, "Digital halftoning," Chapter 10 in Digital Image Processing Methods, E. Dougherty, Ed. New York: Marcel Dekker, 1994

[6] I. J. Cox, M. L. Miller, and J. A. Bloom, Digital Watermarking, Academic Press, 2002.

[7] R. L. de Queiroz, K. Braun and R. Loce, "Detecting spatially varying gray component replacement with application in watermarking printed images," Journal of Electronic Imaging, Vol. 14, No. 3, pp. 033016.1033016.9, Sep. 2005. 
[8] K. T. Knox and S. Wang, "Digital watermarks using stochastic screens - a halftoning watermark," Proceedings of the SPIE International Conference on Storage and Retrieval for Image and Video Databases V, vol. 3022, pp. 310-316, San Jose, CA, US, Feb. 1997.

[9] S. Wang, K. T. Knox, "Embedding digital watermarks in halftone screens," Proceedings of SPIE Security and Watermarking of Multimedia Contents II, Vol. 3971, pp., San Jose, CA, US, Jan. 2000.

[10] M. S. Fu, O. C. Au, "Data hiding in halftone image by pixel toggling," Proceedings of SPIE Security and Watermarking of Multimedia Contents II, Vol. 3971, pp. , San Jose, CA, US, Jan. 2000.

[11] Z. Baharav and D. Shaked, "Watermarking of digital halftones," Journal of Electronic Imaging, Vol. 3657, pp. 307-313, Jan. 1999.

[12] G. Sharma, R. P. Loce, S. J. Harrington, and Y. Zhang, "Illuminant multiplexed imaging: GCR and special effects," Proc. IS\&T/SID Eleventh Color Imaging Conference: Color Science, Systems and Applications, Scottsdale, AZ, pp 266-271, Nov. 2003.

[13] N. D.-Venkata, J. Yen, V. Monga, and B. L. Evans, "Hardcopy image barcodes via block-error diffusion," IEEE Trans. on Image Processing, Vol. 14, no 12, pp. 1977-1989, Dec. 2005.

[14] P. J. Chiang, A. Mikkilineni, E. J. Delp, J. P. Allebach, G. T.-C. Chiu, "Extrinsic signatures embedding and detection in electrophotographic halftone images through laser intensity modulation" Proc. of 22 nd Intl. Conf. on Digital Printing Technologies, NIP 2006, pp. 432-435, 2006.

[15] C. Liu, S. Wang, and B. Xu, "Authenticate your digital prints with Glossmark images," Proc. NIP20: The 20th International Congress on Digital Printing Technologies, Salt Lake City, UT, US, Nov. 2004.

[16] K. R. Rao, and P. Yip, Discrete Cosine Transform, Algorithms, Advantages and Applications, Academic Press, San Diego, CA, 1990.

[17] H. S. Malvar, Signal Processing with Lapped Transforms. Norwood, MA: Artech House, 1992.

[18] R. L. de Queiroz and T. D. Tran, Lapped Transforms for Image Compression, Chap. 5 in The Handbook on Transforms and Data Compression, edited by K. R. Rao and P. Yip, CRC Press, 2001.

[19] R. L. de Queiroz, "Reversible color to gray mapping using subband domain texturization," under review, IEEE Trans. on Image Processing, 2007. 